\title{
MALIGNANT SALIVARY GLAND TUMOURS OF THE NASAL SINUSES AND EXOPHTHALMOS*
}

\author{
BY \\ ALY MORTADA \\ Department of Ophthalmology, Faculty of Medicine Cairo University, Egypt
}

Tumours of the ethmoid, maxillary, frontal, and sphenoid air sinuses usually manifest themselves by proptosis. They may take the form of osteoma, chondroma, osteoclastoma, fibroma, myxoma, angioma, psammoma, endothelioma, carcinoma, or sarcoma (Duke-Elder, 1952). The mucous membrane of these sinuses contains not only epithelial and fibro-adenoid layers but also a layer of mucous and serous glands, a part of the accessory salivary glands which are also present in the palate, lips, tongue, gums, tonsillar fossa, pharynx, larynx, trachea, and nose.

Such accessory salivary glands may give rise to tumours of the same nature as salivary and lacrimal gland epithelial neoplasms. These may be mixed gland tumours or carcinomata. Mixed gland tumours have also been reported in the eyebrow (Gerlach, 1929), eyelids (Wilson, 1938), and lacrimal sac (McCool, 1939; White, Michaelson, and Heggie, 1938). Tumours developing from the glandular elements of the mucous membrane of the nose and paranasal sinuses constitute only about 10 per cent. of the whole group of mixed salivary tumours (Reese, 1951).

Malignant lacrimal or salivary gland tumours may take the form of malignant mixed gland tumour, adeno-carcinoma, or squamous cell, undifferentiated, or adenoid-cystic carcinoma (Hogan and Zimmerman, 1962). Such tumours of the accessory nasal sinuses causing proptosis have been reported so rarely in the ophthalmic literature, that the three following are of great interest.

\section{Case Reports}

The general health of the three patients to be described was moderate. The blood pressure, blood total and differential count, urine, pulse, and temperature were normal, and the blood Wassermann reaction negative. Medical examination showed the chest and abdomen to be normal. Beside malignant enlarged cervical glands in Cases 2 and 3 there were no enlarged lymph glands in the body. X rays of the chest and bones did not show any metastases. 
Case 1, a 20-year-old male (Fig. 1) complained of right proptosis of 2 weeks' duration $\overline{\overline{3}}$ A tumour had been removed from the right cheek 2 months before, and had proved on. histopathological examination to be a pleomorphic adeno-carcinoma. There was swellin $\overrightarrow{F^{2}}$ of the right maxillary region, and at the lower edge of the mandible was seen the scar of the incision through which the cheek tumour had been removed.

The right eye showed an upward pulsating proptosis of $2 \cdot 2 \mathrm{~cm}$. Hertel (compared wit $\frac{{ }_{7}}{5}$ $1.5 \mathrm{~cm}$. on the left side), conjunctival chemosis, limitation of the ocular movements in at directions, and a dilated sluggish pupil. The fundus showed optic atrophy and the ey\& was quite blind. A palpable hard mass was present below the globe.

The left eye was normal, with a normal fundus, and visual acuity 6/9.

Rhinological examination showed an opacity of the right maxillary antrum, and hypertrophic rhinitis, and the hard palate was depressed on the right side. Trismus prevented palpation of the nasopharynx. There were no enlarged cervical glands. Arteriography
showed no ophthalmic or internal carotid aneurysm.

A postero-anterior skull $x$ ray (Fig. 2) showed increased right orbital soft tissue density and diffuse opacity of the right maxillary antrum, with evidence of destruction of its lateral and orbital walls. Right and left oblique skull $x$ rays showed normal optic canals

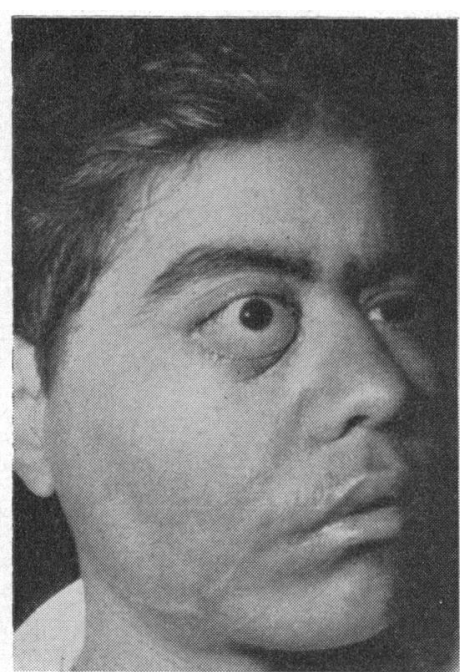

Fig. 1.-Case 1, malignant mixed salivary gland tumour of right maxillary antrum of 2 months' duration in a male aged 20 years. Note right proptosis, swelling of maxillary region, and scar of incision through which the extension to the cheek was removed.

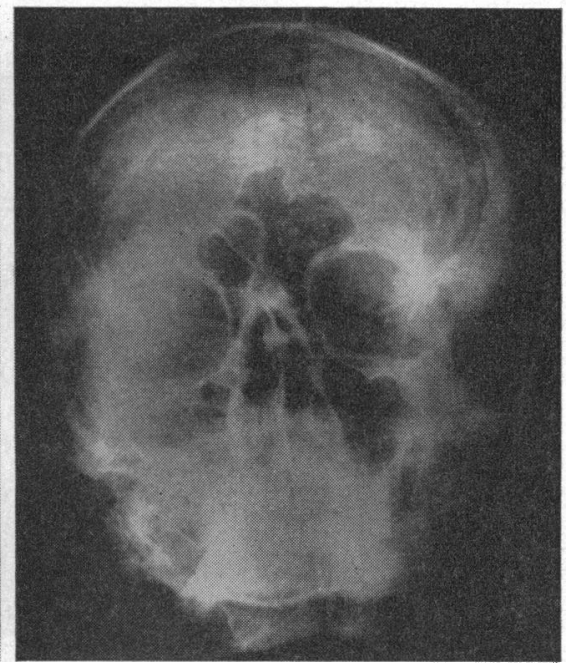

FIG. 2.-Case 1, postero-anterior skull $x$ ray, showing increased right orbital soft tissue density, diffuse opacity of right maxillary antrum, and evidence of destruction of its lateral and orbital walls.

In one week the proptosis became severe (Fig. 3, opposite). Orbital exploration showed that the orbital surface of the frontal process of the maxilla, and the orbital floor were destroyed. A partly hard and partly soft tumour was emerging from the maxillare antrum to occupy the lower and outer parts of the orbit.

The tumour was removed and was found to be non-encapsulated. Histopathologica? examination of the tumour tissue showed, beside acini and nests of epithelial cells in myxomatoid matrix, areas of malignant epitheliomatous nature (Fig. 4, opposite). Th迤 picture was consistent with that of a malignant mixed salivary tumour. 


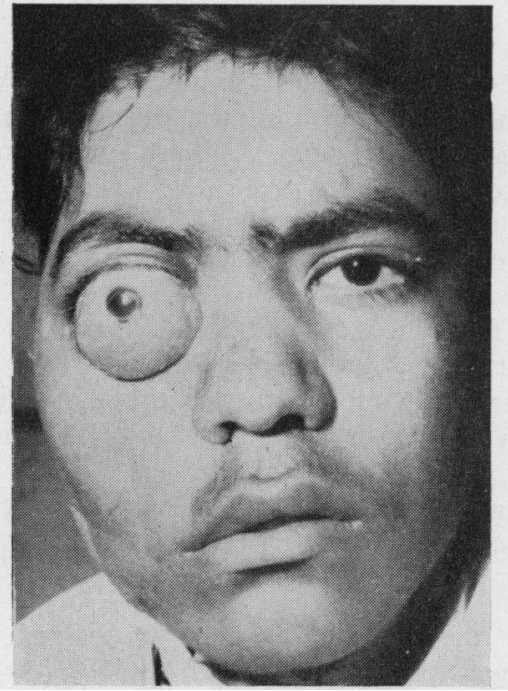

Fig. 3.-Case 1, showing rapid progress of proptosis in one week.

Although the patient was given penicillin and streptomycin injections, 4 days after the operation the maxillary sinus infection extended to the cheek giving rise to a huge abscess (Fig. 5) which was incised and drained.

Case 2, a 40-year old male (Fig. 6) complained of left proptosis and nasal block for 9 months. A rhinologist had removed an ethmoid tumour 4 months before through a

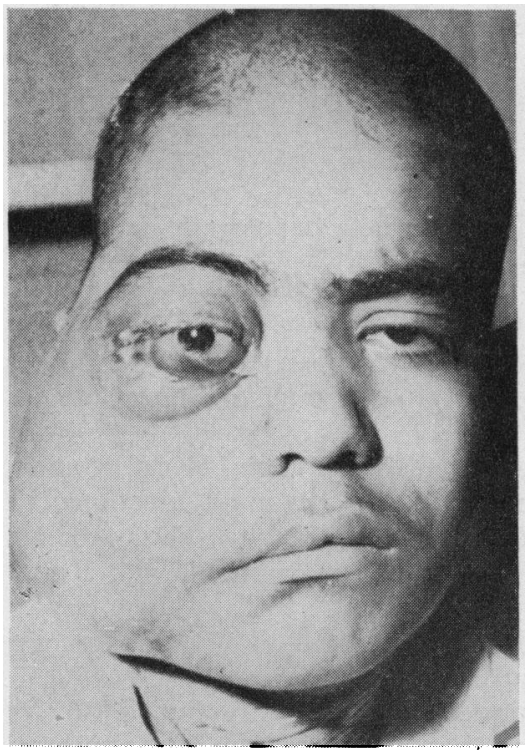

FIG. 5.-Case 1, 4 days after orbital exploration. The maxillary sinus infection extended to the cheek giving rise to a huge abscess.

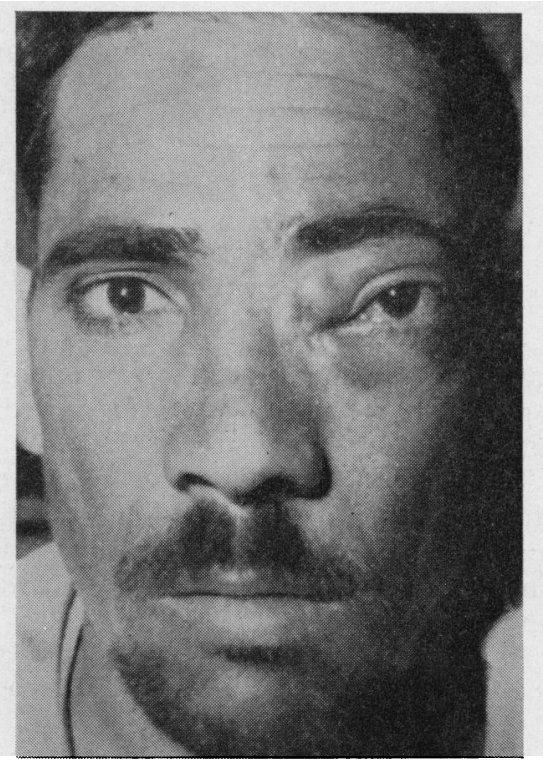

Fig. 6.-Case 2, recurrent salivary gland adenocarcinoma of left ethmoid sinus causing proptosis in a male aged 40 years. 
Moore's incision, but after 2 months the tumour recurred and the proptosis became mo severe.

The left eye showed an outward proptosis of $2.5 \mathrm{~cm}$. Hertel (as compared with $1.6 \mathrm{~cm}$. on the right side), oedema of the lids, and limitation of the ocular movements in agll directions. The fundus was normal and the visual acuity 6/24. A hard orbital mass $1 \times 1 \mathrm{~cm}$. was felt between the globe and the nose.

The right eye was normal, with a normal fundus, and visual acuity $6 / 6$.

Rhinological examination showed a tumour on the left side of the nasal cavity malignant enlarged left cervical glands.

A postero-anterior skull $x$ ray with a $20^{\circ}$ tube tilt (Fig. 7) showed diffuse opacity of the left maxillary antrum. The nasal septum was attenuated and displaced to the right. soft tumour shadow affecting the left ethmoid sinus extended to the left orbit, maxillafy sinus, and nose. The left medial orbital margin was destroyed. Right and left oblique skull $x$ rays showed normal optic canals.

The tumour was removed and found to be non-encapsulated. A histopathologicâ examination of the orbital extension of the tumour showed the picture of an adeng? carcinoma (Fig. 8).

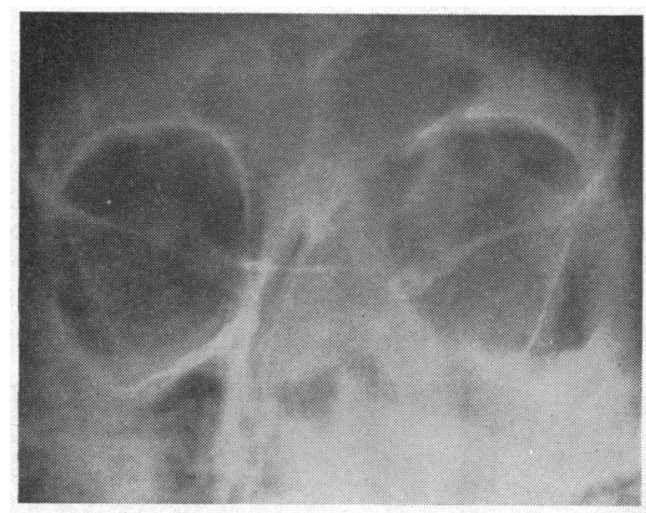

Fig. 7.-Case 2, postero-anterior skull $x$ ray, showing soft tissue tumour shadow affecting the left ethmoid sinus, orbit, maxillary sinus, and nose. Note destruction of left medial orbital margin and deviation of nasal septum to the right.

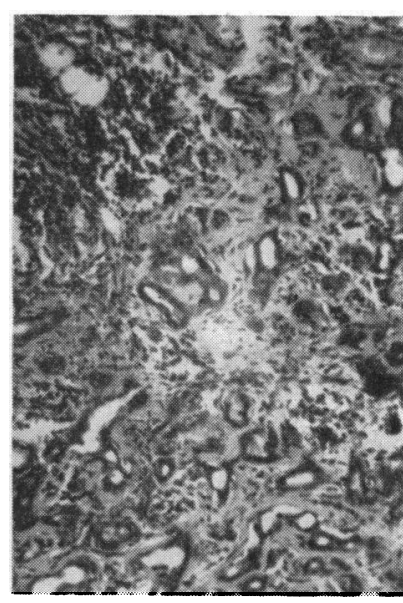

FIG. 8.-Case 2, section of adeno-carcinoma of accessory salivary glands of left ethmoid sinus. $\times 75$.

Case 3, a 35-year-old woman (Fig. 9, opposite), had a right proptosis of 5 months' duration A right orbital tumour had been removed one month before through an upper lid incision but the tumour had recurred.

The right eye showed downward and outward proptosis of $2.5 \mathrm{~cm}$. Hertel (comparê with $1.6 \mathrm{~cm}$. on the left side) and limitation of ocular movements in all directions. Tht fundus showed optic atrophy with no vision. A hard nodular mass was felt in the uppes inner part of the orbit extending backwards. The left eye was normal, with a normegt fundus, and visual acuity 6/9.

Rhinological examination revealed opacity of the left ethmoid and frontal air sinuse The left cervical lymph glands were large and hard.

A postero-anterior skull $x$ ray (Fig. 10, opposite) showed destruction of the right medial orbital wall affecting the right ethmoid and frontal air sinuses. The appearances were those of a right ethmoid malignant growth invading the orbital cavity and front 1 sinus. Right and left oblique skull $x$ rays showed normal optic canals. 

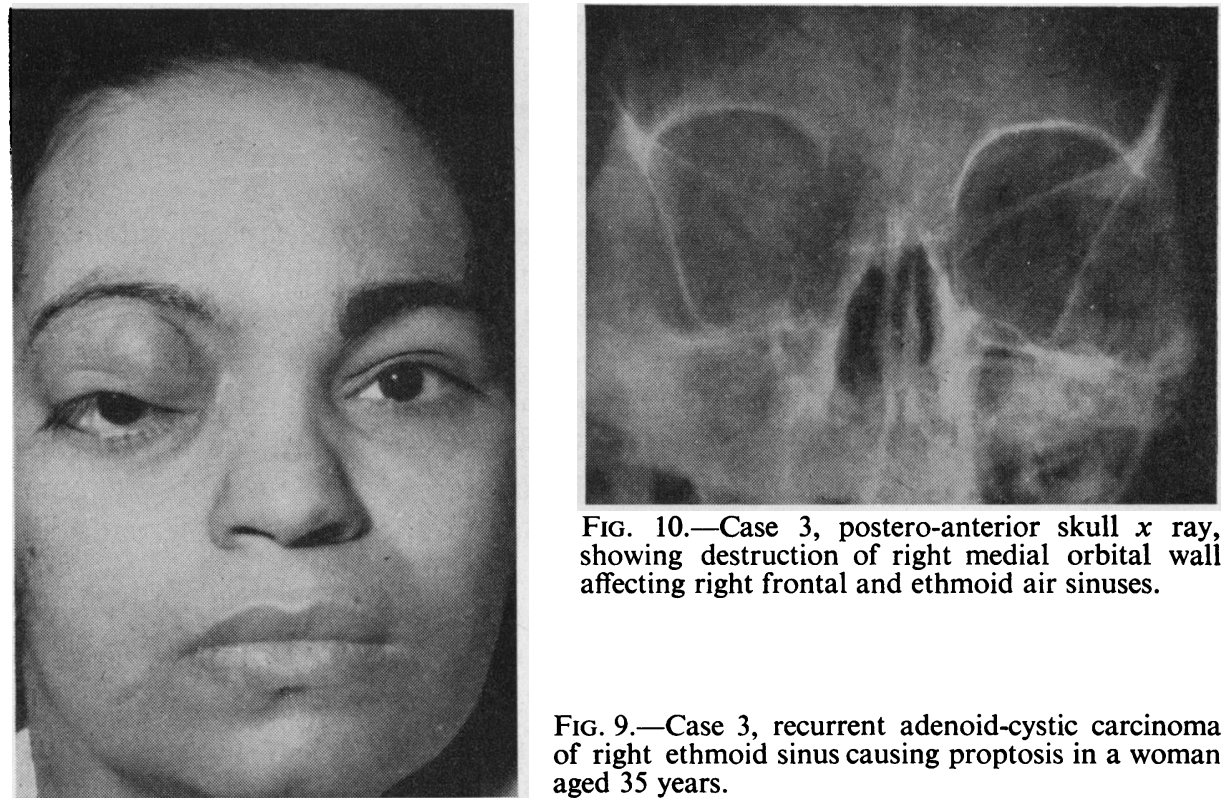

FIG. 10.-Case 3, postero-anterior skull $x$ ray, showing destruction of right medial orbital wall affecting right frontal and ethmoid air sinuses.

Orbital exploration showed a non-encapsulated tumour. Histopathological examination showed tightly packed epithelial cells containing hyperchromatic nuclei and scanty cytoplasm. The epithelial aggregations contained cystic foci of various sizes and shapes containing degenerated epithelial cells and mucin (Figs 11 and 12). The picture was consistent with that of an adenoid cystic carcinoma.

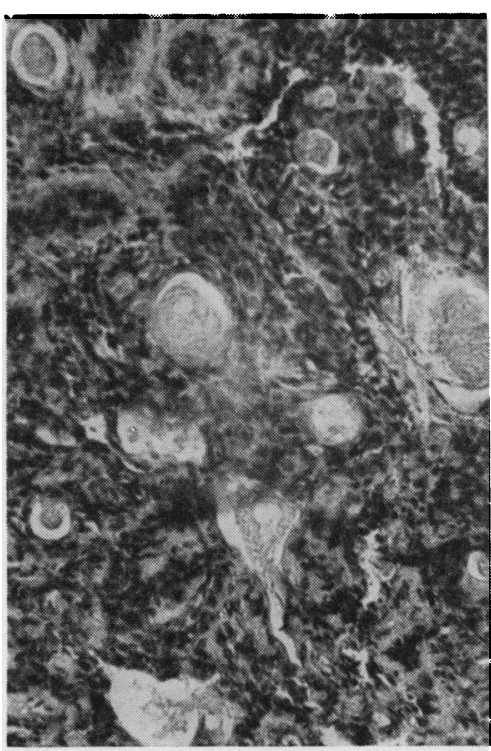

FIG. 11.-Case 3, section of tumour, showing aggregations of epithelial cells with small cystic areas containing mucin. $\times 96$.

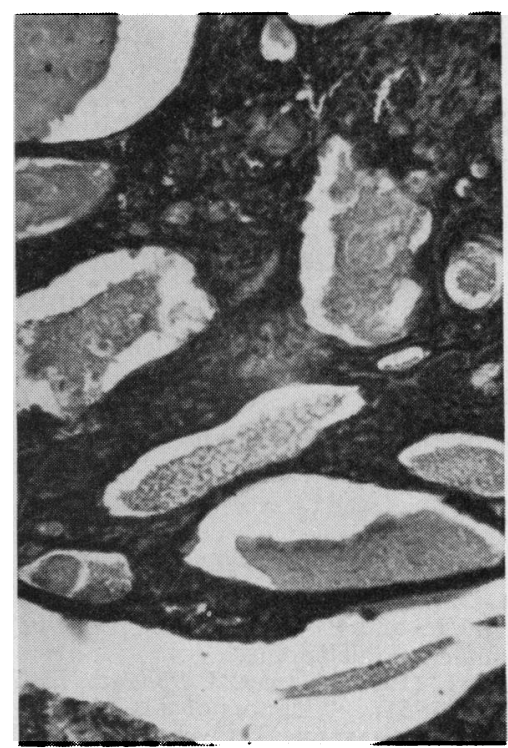

FIG. 12.-Case 3, section of tumour, showing large cystic foci containing mucin. $\times 96$ 
The three cases were referred to the rhinologist for treatment. Attempt to remove the tumours were followed by $x$ ray treatment, but in all cases the tumours recurred and the prognosis appeared poor.

\section{Discussion}

In the orbit, mixed gland tumours, adeno-carcinomata, and adenoido cystic carcinomata may not only arise from the lacrimal and accessory lacrio mal glands but may also occur as an orbital extension of accessory salivary gland tumours from the mucous membrane of the nose and nasal sinuses.

In a series of nasal sinus tumours extending to the orbit and giving rise to proptosis, beside the described three cases there were 5 undifferentiated care. cinomata and 5 osteomata of the ethmoido-frontal sinuses, one fibromyxoma of the frontal sinus, three reticulin cell sarcomata and three undifferentiated carcinomata of the maxillary antrum.

In the three cases described, a postero-anterior skull $x$ ray with $20^{\circ}$ tube्ध tilt showed the site of the affected sinus. Only orbital exploration, biopsy, and histopathological examination revealed the nature of the lesion.

In Case 1, the maxillary sinus malignant mixed salivary gland tumour first destroyed the lateral wall of the maxillary antrum and presented as $\overrightarrow{8}$ cheek tumour, and then destroyed the orbital wall of the sinus and presented as a vascular orbital tumour with pulsating exophthalmos. During orbitad exploration, palpation of the destroyed roof and lateral walls of the maxillary sinus was followed by a spread of infection from the affected sinus to the cheek and caused a huge abscess.

\section{Summary}

(1) In a series of 130 histopathologically diagnosed orbital tumours therêf were three malignant recurrent epithelial salivary gland tumours of the nasa: sinuses presenting with proptosis.

(2) Case 1 showed a maxillary sinus malignant mixed salivary tumoug. presenting as pulsating exophthalmos. The other two cases were due to adeno-carcinoma and adenoid-cystic carcinoma of the ethmoid sinuses.

(3) These are the first three cases of such tumours with orbital extension? causing proptosis to be reported from Egypt.

\section{REFERENCES}

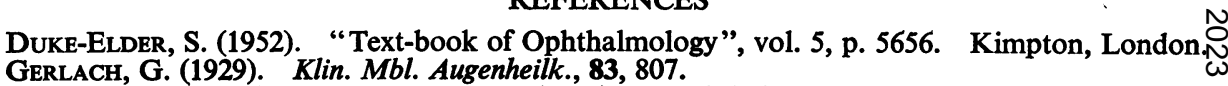

Hogan, M. J., and Zimmerman, L. E. (1962). "Ophthalmic Pathology", 2nd ed., p. 758 Saunders, Philadelphia.

MCCoOL, J. L. (1939). Amer. J. Ophthal., 22, 734.

REESE, A. B. (1951). "Tumors of the Eye", p. 478. Hoeber, New York.

WhITE, J. P., Michaelson, I. C., and HeGGIE, J. F. (1938). Trans. ophthal. Soc. U.K., 58, 159:?

Wilson, R. P. (1938). Ann. Rep. Memorial ophthal. Lab., Giza, Cairo, vol. 13, p. 41. (Cited by Duke-Elder, 1952, p. 5071.) 